\title{
A look at the cosmic ray anisotropy with the nonlocal relativistic transport approach
}

\section{A. D. Erlykin}

P.N.Lebedev Physical Institute, Moscow, Russia

Physics Department, Durham University, Durham, UK

E-mail: erlykin@sci.lebedev.ru

\section{R. T. Sibatov}

Ulyanovsk State University, Ulyanovsk, Russia

E-mail: ren_sibabk.ru

\section{V. Uchaikin}

Ulyanovsk State University, Ulyanovsk, Russia

E-mail: vuchaikin@gmail.com

\section{A. W. Wolfendale}

Physics Department, Durham University, Durham, UK

E-mail: a.w.wolfendale@durham.ac.uk

\begin{abstract}
The Cosmic Ray anisotropy is a key element in the quest to find the origin of the enigmatic particles. A well known problem is that, although most of the likely sources are in the Inner Galaxy, the direction from which the lowest energy particles (less than about $1 \mathrm{PeV}$ ) come is largely from the Outer Galaxy. We show that this can be understood taking into account a possible reflection of charged particles by 'walls' in the Interstellar Medium or/and as a temporary phenomenon after the shock wave from the supernova explosion passed the Earth. This effect is too subtle to be explained by an ordinary diffusion theory and becomes apparent within the frames of the non-local relativistic transport theory, which involves conceptions of free motion velocity and path lengths with probability distributions of non-exponential type taken for a turbulent interstellar medium.
\end{abstract}

The 34th International Cosmic Ray Conference,

30 July- 6 August, 2015

The Hague, The Netherlands

\footnotetext{
${ }^{*}$ Speaker.
} 


\section{Introduction}

The problem of the nature of the sources of the observed Cosmic Rays (CR) is well known: irregularities in the magnetic fields in the Interstellar Medium (ISM) cause the direction of a CR observed at Earth to bear little relation to the direction to its actual source, except for CR of very high energies.

In a recent paper on the anisotropy problem [1] we analysed the problem in detail using all available data. The results confirm the well-known facts that there is a region at energies below $1 \mathrm{PeV}$ which has a phase opposite to expectation and a marked change of the phase in the Right Ascension (RA) plot starting from $1 \mathrm{PeV}$, where the amplitude of the anisotropy drops and then starts to rise again.

Although it is possible to design an 'origin-model' which explains the phenomena it seems rather contrived. A fundamental question is the following: can particles originated from a source in a particular direction be observed at Earth as coming from the opposite direction or generally from any different direction? In particular, can the 'flow' of particles from the Inner Galaxy give an anisotropy pointing to the opposite direction, i.e. to the Outer Galaxy?

\section{On the non-local CR-diffusion model}

The transport of CR in the Galaxy is an extremely complex process dependent on changeable in space and time interstellar medium characteristics which are known to us only in outline. This is why we are forced to use more or less simplified models for description of the process. The standard diffusion model is a very crude approximation which gives only qualitative estimates of only some aspects of the process. Suffice it to say that the diffusion model of CR propagation in space violates the relativistic principle of velocity limitation. In spite of the conventional diffusivity representation $D \propto v l$, the diffusion equation relates to the limit case with the infinite velocity $v$ and zero free path $l$. For this reason, the diffusion model is incapable of describing the CR transport near boundary separating regions with different properties. Trajectories of this process called the Brownian motion are nowhere differentiable which makes them infinitely far from their physical prototypes (Fig. 1,a). In particular, the length of any section of such trajectory between two arbitrary points is infinitely long.

Because of their self-similarity however, the trajectories retain these properties at all scales including arbitrary small ones. Thus, despite the fact that the diffusion equation can be derived from the feasible random walk with a finite velocity by passage to large scales, the way back is closed: the small-scale behavior of the particles holds its Brownian pattern which looks here even more unnatural than on large scales. For these reasons, the diffusion model and their modifications based on the diffusivity concept are not really in a position to give proper particle path lengths and their escape time distributions.

It is well known that already hydrodynamic turbulence deprives the Brownian motion status of a reliable mathematical model, and one could only wonder how long the diffusion ideas can live in the theory of CR transport. Nevertheless, one can say, the classical diffusion model is gradually losing its position. The process has been partially traced in reviews [2, 3]. One can consider these reviews as a relatively young branch of transport theory: relativistic Brownian motion. The 

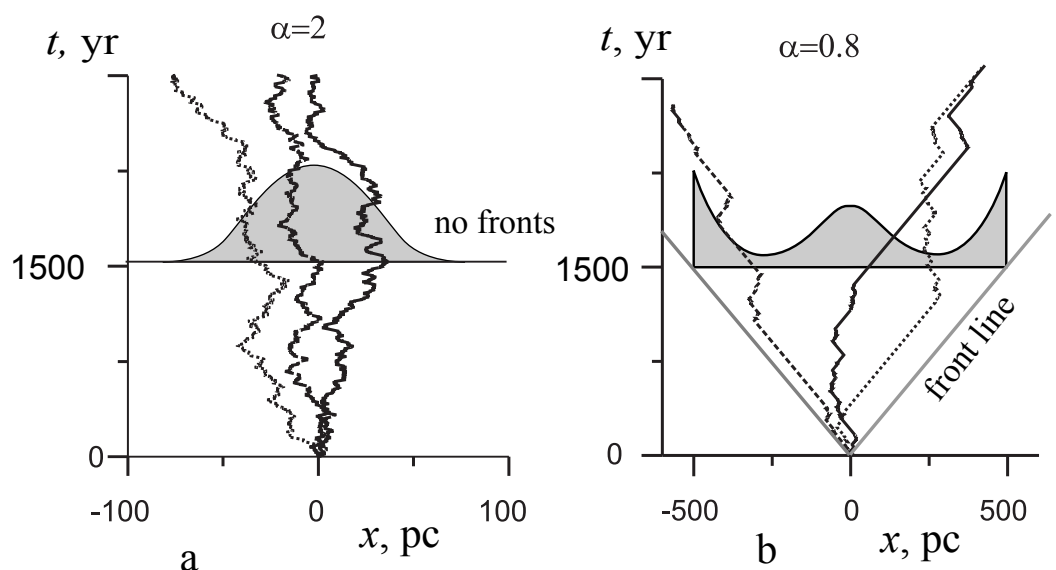

Figure 1: Typical trajectories of random walk with constant velocity for different $\alpha$. The 'front line' corresponds to the speed of light.

idea to get a relativistic analogue of the plain diffusion equation by generalising the link of the latter with the quantum Schrödinger equation was first realized in article [4]. Applying the similar transformation to the Dirac equation, the authors obtained the result in the form of the telegraph equation representing the relativistic Brownian motion with a finite velocity (limited by the speed of light) and including exponentially distributed free paths. A key item of the approach is replacement of the non-relativistic first Fick law

$$
\mathbf{j}(\mathbf{x}, t)=-D \operatorname{grad} n(\mathbf{x}, t)
$$

by its "relativistic" counterpart containing a retarded operator,

$$
\mathbf{j}(\mathbf{x}, t)=-\int_{0}^{t} v^{2} e^{-v^{2} \tau / D} \operatorname{grad} n(\mathbf{x}, t-\tau) d \tau, \quad D=l v / 3
$$

Combining it with the continuity equation yields the telegraph equation derived for onedimensional transport problems half of century earlier [5]:

$$
\frac{\partial n}{\partial t}+\theta \frac{\partial^{2} n}{\partial t^{2}}-D \frac{\partial^{2} n}{\partial x^{2}}=S(x, t),
$$

where $S(x, t)$ is a source term $(S(x, t)=\delta(x) \delta(t)$ for instantaneous point source). More formal mathematical generalization of classical Brownian motion to its relativistic counterpart is performed by Dunkel and Hänggi [6].

However, this equation describes propagation of CRs through a system of mutually independent scatterers, whereas real points of scattering are bounded by magnetic field lines along which the particles fly. This means that free path of CRs particles should be distributed rather according to power type (similarly to other laws in turbulent media) than to exponential one (the latter relates to an ideal gas in the equilibrium state). For this reason, the right hand side of eq. (2.3) is replaced by some non-local (in space-time) operator, averaging it over turbulent fluctuations (see [2]). As a result, the exponential law changes to the power type Lévy distribution $p_{\xi}(x) \propto x^{-\alpha-1}$ with an exponent $\alpha \in(0,2]$ connected with the fractal dimension of the ISM. All these improvements have 
drastically changed the picture making it closer to a real transport process (Fig. 1,b). One can see now the front lines $(x= \pm v t)$, free paths being parallel to one of the lines and having random lengths distributed according to the inverse power law. The latter is characterized by numerous small segments intermitted by long 'Lévy jumps'.

It is worth noting that in earlier articles on anomalous diffusion [7, 8, 9, 10], CR propagation was described in terms of instantaneous 'Lévy jumps' and this analysis was used in [11] to determine the different scattering properties of the Inner and Outer Galaxy. Perfecting this model, we replaced instantaneous spatial jumps by straight lines with a finite velocity of motion along them (that is replaced Levy jumps by Levy flights) [12]. We derived the corresponding integrodifferential equation and extracted from it the following asymptotical 1D-version [12]

$$
\left[\left(\frac{\partial}{\partial t}-v \frac{\partial}{\partial x}\right)^{\alpha}+\left(\frac{\partial}{\partial t}+v \frac{\partial}{\partial x}\right)^{\alpha}\right] n(x, t)=S_{\alpha}(x, t), \quad 0<\alpha<1
$$

where $S_{\alpha}(x, t)$ is a generalized source term $\left(S_{\alpha}(x, t)=\frac{t^{-\alpha}}{2 \Gamma(1-\alpha)}[\delta(x-v t)+\delta(x+v t)]\right.$ for instantaneous point source).

$$
\frac{\partial n(x, t)}{\partial t}-\frac{v c_{\alpha}}{2\langle R\rangle}\left[\left(\frac{\partial}{\partial t}-v \frac{\partial}{\partial x}\right)^{\alpha}+\left(\frac{\partial}{\partial t}+v \frac{\partial}{\partial x}\right)^{\alpha}\right] n(x, t)=S_{\alpha}(x, t), \quad 1<\alpha<2
$$

where $\langle R\rangle$ is the mean free path length, $c_{\alpha}$ a scale constant (see details in [2]).
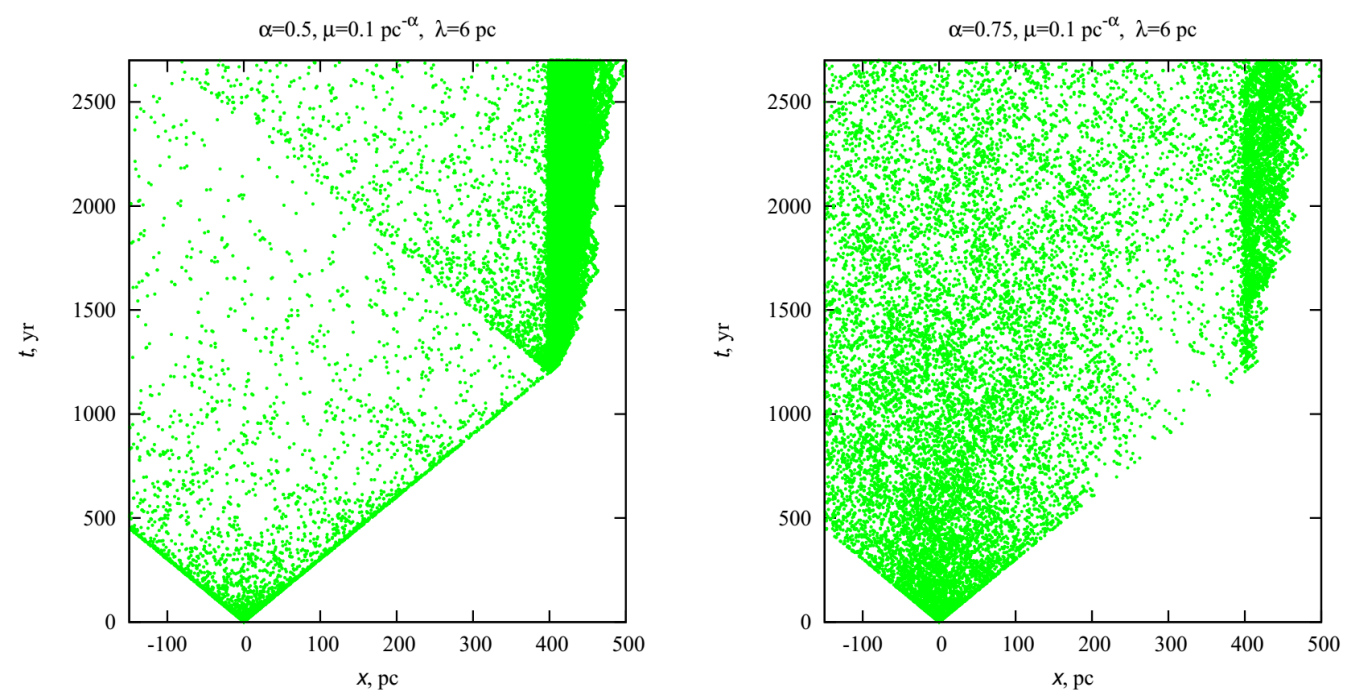

Figure 2: Scattering diagram of random walks in a two-layer medium $(\alpha=0.5$ and 0.75 for $x<400 \mathrm{pc}$ and $\alpha=1.75$ for $x>400 \mathrm{pc}$ ).

\section{Results of the calculations}

Numerical results represented below are calculated by Monte Carlo method and they support the analytical evaluation. Omitting calculation details, we pass directly to a discussion of the results.

We begin searching an answer to question if the inversion of the CR's anisotropy (in the sense formulated above) is generally possible with consideration of a one-dimensional process. The 

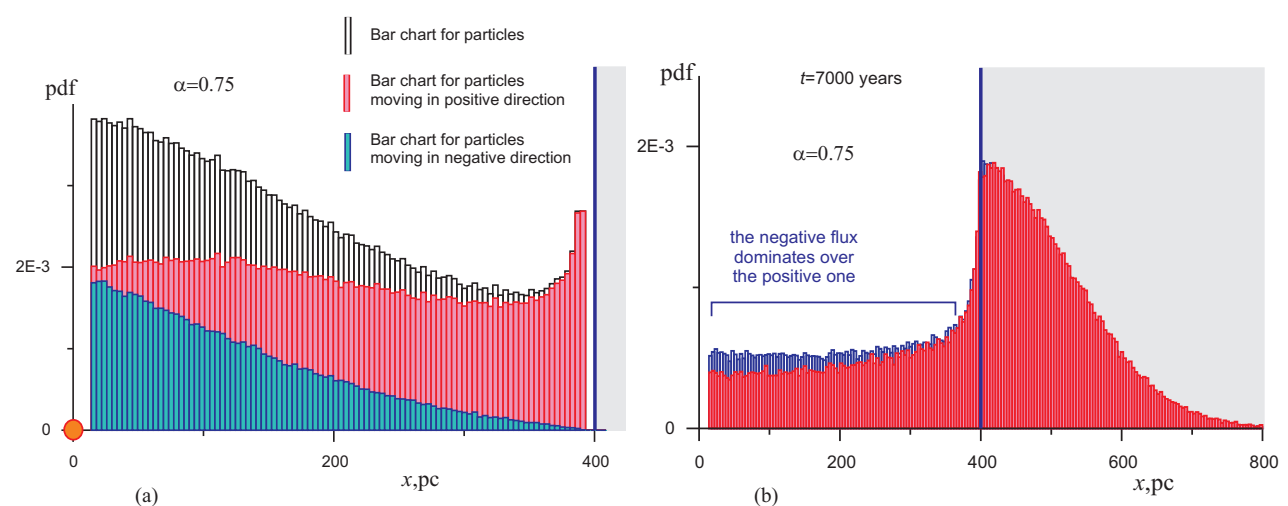

Figure 3: Bar charts for particles moving in positive (red) and negative (blue) directions before (a) and after (b) hitting the boundary, $\alpha=0.75$ for $x<400$ and $\alpha=1.75$ for $x>400$

continuity equation

$$
\frac{\partial n(x, t)}{\partial t}=-\frac{\partial j_{x}(x, t)}{\partial x}
$$

shows that in the stationary case, when $n(x, t)=n(x), j_{x}(x)$ doesn't depend on $x$ and consequently has the same sign (positive to the right of the source and negative on the left). In other words, the flux is directed from the local source. This conclusion is valid for heterogeneous media too (if not, the particles should be accumulated in some region and the process will be time-dependent).

In case of a time-dependent source, the ordinary diffusion model gives the similar result, because the diffusion equation is a parabolic one and has no wave-solutions which could reverse concentration gradient. However, a more realistic telegraph equation (2.3) is hyperbolic, it demonstrates such feature of the wave motion as the existence of a front splash and a retardation. But this equation relates to a uniform medium when free paths are distributed according to exponential law. The turbulent character is taken into account by its fractional counterpart following from (2.4-2.5).

We have solved this equation both analytically and numerically (by means of Monte Carlo method). The latter is especially effective in case of particles passing through a boundary separating two regions with different properties. As our calculations show, the fractional generalization also reveals the appearance of a weak short-term current inversion after the front flash. This weak phenomenon becomes more significant near the boundary between two domains with essentially different properties and long free paths in one of them (especially, when $\alpha<1$ ). Some preliminary results obtained within the frames of one-dimensional (forward and backward) walk model, are presented in Figs. 2-5.

The results are obtained for the case when a point short-time source placed at the origin and emits particles moving along $x$-axis. We observe the forward and backward currents and obtain the anisotropy in the usual way. The inversion of isotropy appears only when we combine a two-layer medium with a finite velocity of motion. In this case, we really observe negative isotropy after the short thick front layer of particles passed by, which give enough number of back-scattered particles close to the boundary. It is remarkable that the inversion of current is observed on the source side near the boundary, when the front of the incident packet is large enough (Fig. 2). This phenomenon can be interpreted as a stochastic reflection of the packet front from the dense medium. 

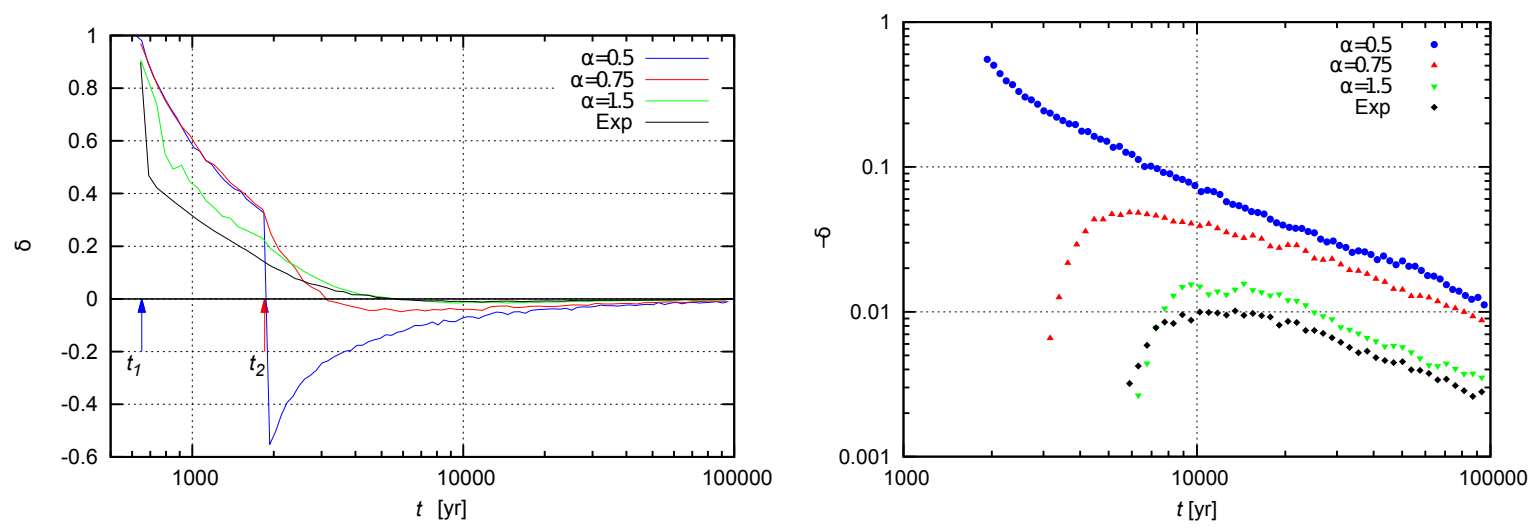

Figure 4: Left panel: The family of anisotropy time-dependence $\delta(t)$ for various values of $\alpha$. Right panel: Function $-\delta(t)$ in the log-log scale. The observation point is to the left of the boundary (that is, in 1medium). Distances: source-observer $R=200 \mathrm{pc}$ and source-boundary $a=400 \mathrm{pc}$. Random free paths are distributed according to a power law with exponents $\alpha$ in 1-medium and $\beta$ in 2-medium $(\beta=1.9$; mean path in 2-medium $\lambda_{2}=0.3 \mathrm{pc}$ ). First-particles front reaches the detector at the time point $t_{1}=R / v$ than it reaches boundary, dives into 2-medium, diffuses there, partially returns through the boundary and occurs the observer point after $t_{2}=[a+(a-R)] / v$. Label 'Exp' corresponds to the case of exponential distribution of free path lengths.
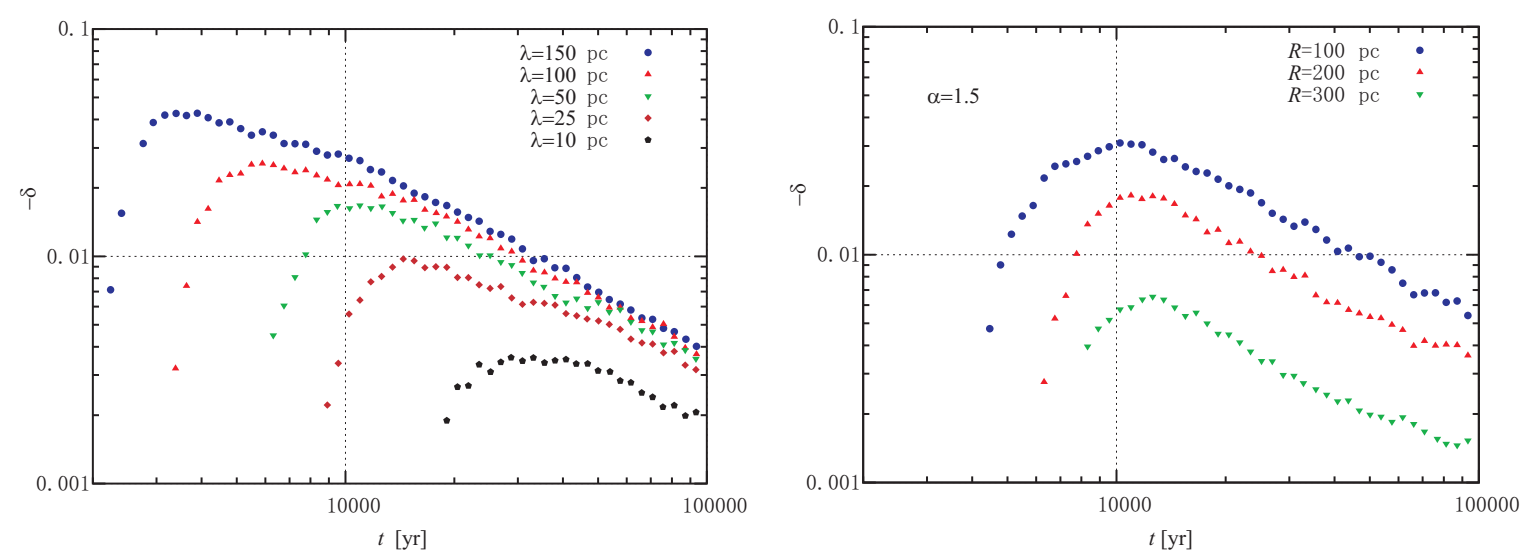

Figure 5: Left panel: Anisotropy $\delta(t)$ for various values of mean path $\lambda$ in the first layer $(\alpha=1.5)$. Right panel: $\delta(t)$ for various detector positions in the first layer ( $R$ is the source-detector distance). The observation point is to the left of the boundary (that is, in 1-medium). The source-boundary distance is equal to $a=400 \mathrm{pc}$. Random free paths are distributed according to a power law with exponents $\alpha=1.5$ in 1 -medium and $\beta=1.9$ in 2-medium ( $\beta=1.9$; mean path in 2-medium $\lambda_{2}=0.3 \mathrm{pc}$ ).

Calculated results of the time-dependent anisotropy are presented in Fig. 4 and 5 for different values of $\alpha$ and different detector positions. The source-boundary distance $a=400$ pc. Random free paths are distributed according to the inverse power law with exponent $\alpha$. The 'first-particles front' reaches the boundary, dives into the second medium, diffuses there, partially returns through the boundary and appears at the observer after $t_{\text {front }}=(a+(a-R)) / v$. In case of $R=200 \mathrm{pc}$, $t_{\text {front }} \approx 1957 \mathrm{yr}$. 


\section{Conclusion}

We argue that the presence of local regions of reduced diffusion coefficient (D) can seriously perturb the directions from which CR arrive from a specific source which generated CR for a limited period. For example, a close-by region of reduced D can "store" particles such that more source-particles come from the anti-source direction than the reverse. The location of the Solar System in the Local Bubble might satisfy this condition.

The numerical investigation undertaken in this work shows that the anisotropy inversion phenomenon can be explained theoretically, although one has to take into account the following circumstances.

1. This phenomenon usually does not take place in the case of stationary (time-independent) transport.

2. The ordinary diffusion theory cannot catch this phenomenon because it is not in a position to describe the front splash: a diffusion packet is instantaneously spread around all space, breaking the relativistic principle.

3. The more advanced transport theory reveals the reality of such anisotropy inversion mechanism: the most auspicious conditions for the phenomenon appear after the front splash passed through the boundary of two domains in the direction of the more dense one.

Attempting to give a more clear explanation of the anisotropy inversion phenomenon, we suggest to imagine what happens when a finite duration pulse of light falls on a perpendicular plane. If the latter is an absorber, the pulse is not reflected: it sticks in the absorber as the ordinary diffusion predicts. If the plane is of a mirror type, then the pulse is reflected with inverted form: the growing front part (moving now in negative direction) is superposed on the fading tail of the pulse (moving yet in positive direction), so the sum may become negative. This process is described by a plane kinematics. In reality, the particles penetrate through the plane into small deep of the second medium and most of them are finally reflected. This is a little bit more complex process described by the telegraph equation, so we meet some intermediate case, but our calculation showed that the phenomenon of anisotropy inversion continues to take place.

Application of the above techniques to the case of the Solar System, i.e. estimating the expected anisotropy locally, is difficult for two reasons.

(i) The topography of the local ISM is not (yet) well known.

(ii) The time profile of the CR intensity from the various local sources is similarly uncertain.

However, some progress can be made in a rather general way. Concerning (i) two major features of the local ISM are apparent: the Local Bubble and the Local Fluff. The Local Bubble [13] is a cavity of the reduced density of the ISM around the Solar System. It has an approximate hourglass shape and its boundary towards the Outer Galaxy is situated at a distance of about $300 \mathrm{pc}$ from the Sun. The mean density inside the Bubble is about $0.05 \mathrm{~cm}^{-3}$. At larger distances the density comes back to its mean ISM value of $0.5 \mathrm{~cm}^{-3}$. The boundary between regions of low and high ISM density can be regarded as 'the wall' for CR populating the interiors of the Local Bubble.

However, the low ISM density inside the Bubble is not perfectly uniform. It contains numerous relatively small cloudlets with different sizes, shapes, densities, temperatures and other characteristics. At the present time the Solar System enters one of such cloudlets, usualy called Local Interstellar Cloud or Local Fluff (LF) [14]. It has approximate diameter of 10pc, density of 
$0.1 \mathrm{~cm}^{-3}$ and temperature of $6000^{\circ} \mathrm{K}$. Measurements made by the Voyager- 2 mission outside the Solar System have found that LF is highly magnetized with a mean field of about 4-5 $\mu G$ [15]. It is clear that due to its higher density and magnetic field the diffusion inside the LF is slower than in the surrounding Local Bubble. Thus, inspite of all the deficiencies in our knowledge of the ISM topography it can be assumed that in the vicinity of the Solar System there are conditions which can create the inversion of the CR anisotropy.

Acknowledgment. AWW and ADE thank the Kohn Foundation for financial support of this work. VVU and RTS thank the Russian Foundation for Basic Research (project 13-01-00585).

\section{References}

[1] A. Erlykin, A. Wolfendale, The role of the galactic halo and the single source in the formation of the cosmic ray anisotropy. Astroparticle Physics 60 (2015) 86-91.

[2] V.V. Uchaikin, Fractional phenomenology of cosmic ray anomalous diffusion. Physics-Uspekhi 56, 11 (2013) 1074-1119.

[3] V.V. Uchaikin, Nonlocal models of cosmic ray transport in the Galaxy. Journal of Applied Mathematics and Physics 3, 02 (2015) 181-194.

[4] B. Gaveau, T. Jacobson, M. Kac, L. Schulman, Relativistic extension of the analogy between quantum mechanics and Brownian motion. Physical Review Letters 53, 5 (1984) 419.

[5] V. Fock. Naherungsmethode zur Losung des quantenmechanischen Mehrkorperproblems. Zeitschrift Physik 61 (1930), 126-148.

[6] J. Dunkel, P. Hänggi, Relativistic Brownian motion. Physics Reports 471, 1 (2009) 1-73.

[7] B.R. Ragot, J.G. Kirk, Anomalous transport of cosmic ray electrons. Astron. Astrophys. 327 (1997) 432.

[8] A. Lagutin, Y. Nikulin, V. Uchaikin, The "knee" in the primary cosmic ray spectrum as consequence of the anomalous diffusion of the particles in the fractal interstellar medium. Nuclear Physics B-Proceedings Supplements 97, 1 (2001) 267.

[9] A. Lagutin, V. Uchaikin, Anomalous diffusion equation: Application to cosmic ray transport. Nuclear Instruments and Methods in Physics Research Section B: Beam Interactions with Materials and Atoms 201, 1 (2003) 212.

[10] A. Erlykin, A. Lagutin, A.W. Wolfendale, Properties of the interstellar medium and the propagation of cosmic rays in the Galaxy. Astroparticle Physics 19, 3 (2003) 351.

[11] A. Erlykin, A. Wolfendale, Cosmic ray anisotropies to 5 PeV. Journal of Cosmology and Astroparticle Physics 04 (2013) 006.

[12] V.V. Uchaikin, On the fractional derivative model of the transport of cosmic rays in the Galaxy. JETP letters 91, 3 (2010) 105.

[13] D.P. Cox, R.-J. Reynolds, The Local Interstellar Medium. Annual Review of Astronomy and Astrophysics 25 (1987) 303.

[14] P.C. Frisch, The Local Bubble, Local Fluff and Heliosphere (1997) [arxiv:astro-ph/9710141].

[15] M. Other, F. Alouani Bibi, G. Toth, et al., A strong, highly tilted interstellar magnetic field near the Solar System. Nature 25 (2009) 1036. 